\title{
Correction to: Positron Emission Tomography in Drug Development
}

Frans van den Berg and Eugenii A. (Ilan) Rabiner

\section{Correction to: R. Schreiber (ed.), Modern CNS Drug Discovery, https://doi.org/10.1007/978-3-030-62351-7_11}

The original version of Chapter 11 was inadvertently published without the first name of the co-author. The name "A. (Ilan) Rabiner" has now been corrected to "Eugenii A. (Ilan) Rabiner". 